\title{
ARTÍCULOS
}

Recibido 03.07.2013. Aprobado 25.11.2013

Evaluado por el sistema double blind review. Editor científico: Jairo Eduardo Borges-Andrade

DOI: http://dx.doi.org/10.1590/So034-759020140607

\section{ANTECEDENTES DE LAS INTENCIONES DE ABANDONO EN COOPERATIVAS COLOMBIANAS}

\author{
Antecedentes das intenções da rotatividade nas cooperativas colombianas \\ Antecedents of turnover intentions in Colombian cooperatives
}

\begin{abstract}
RESUMEN
El artículo describe un estudio cuantitativo sobre las intenciones de dejar la organización por parte de los trabajadores de algunas cooperativas colombianas. Las 3 empresas participantes fueron creadas con el fin de dar empleo a la población femenina local golpeada por la violencia política. Este tipo de empresas, de estructura cooperativista y organizadas alrededor de una misión social fundamental, enfrentan problemas de reclutamiento y retención de personal. 332 trabajadores participaron en la investigación. Para el análisis de datos se utilizó la metodología SEM. Los resultados indican que el intercambio líder-miembro tiene un efecto negativo indirecto sobre las intenciones de dejar la organización. En congruencia con la investigación precedente, esta relación aparece mediada por el apoyo organizacional percibido. Se encontró que la implicación en el trabajo también mediaba el efecto de la relación líder-miembro sobre las intenciones de dejar la organización. Algunas limitaciones del estudio, consideraciones prácticas/teóricas son presentadas al final del artículo.
\end{abstract}

PALABRAS-CLAVE | Intenciones de dejar la organización, cooperativas, Colombia, intercambio líder-miembro.

\section{RESUMO}

$O$ artigo descreve um estudo quantitativo sobre as intenções da rotatividade por parte dos trabalhadores de algumas cooperativas colombianas. As três empresas participantes foram criadas com o objetivo de fornecer emprego para a população feminina local vitimada pela violência política. Este tipo de empresa, de estrutura cooperativista e organizada ao redor de uma missão social fundamental, enfrenta problemas de recrutamento e retenção de pessoal. 332 trabalhadores participaram da pesquisa. Para a análise de dados, a metodologia foi a Modelagem por Equações Estruturais. Os resultados indicam que o intercâmbio líder-membro possui um efeito negativo indireto sobre as intenções da rotatividade. Em congruência com pesquisa precedente, esta relação parece mediada pelo apoio organizacional percebido. Descobriu-se que a inserção no trabalho também mediava o efeito da relação líder-membro sobre as intenções da rotatividade. Algumas limitações do estudo e considerações práticas e teóricas são apresentadas no final do artigo.

JUAN PABLO ROMÁN-CALDERÓN

jromanca@eafit.edu.co Profesor del Departamento de Psicología, Universidad EAFIT Medellín, Colombia

\section{ADALGISA BATTISTELLI}

adalgisa.battistelli@u-bordeaux.fr Profesor del Departamento de Psicología, Université Bordeaux Bordeaux, France

\section{MARIO VARGAS-SAENZ}

mvargas@eafit.edu.co

Director de la EAFIT Social, Universidad

EAFIT - Medellín, Colombia
PALAVRAS-CHAVE / Rotatividade, cooperativas, Colômbia, intercâmbio líder-membro.

\begin{abstract}
This paper presents a study on the intentions to quit of employees working in Colombian cooperatives. These enterprises were created to include woman affected by political violence into the labor market. According to some scholars, cooperative enterprises driven by a fundamental social mission face retention and recruitment problems. 332 workers participated in the survey. Structural Equation Modeling was used for data analyses. The findings suggest that within this type of enterprise, leader-member exchange has a negative indirect effect on turnover intentions. Consistent with previous research, this relationship is mediated by perceived organizational support. Moreover, the relationship between leader-member exchange and turnover intentions is also mediated by job-embeddedness. The limitations of the study as well as practical and theoretical implications are presented at the end of the paper.
\end{abstract}

KEYWORDS / Turnover, cooperatives, Colombia, Leader-member exchange. 


\section{INTRODUCCIÓN}

La mayor parte de la investigación sobre los recursos humanos de las organizaciones ha sido desarrollada en entidades con ánimo de lucro. Una segunda vertiente de estudios en esta área del conocimiento se ha dedicado a estudiar los lazos que los voluntarios establecen con organizaciones con fines sociales y/o con sus trabajos. Contrariamente, existe poca investigación acerca de la relación que establecen los trabajadores con aquellas organizaciones que se encuentran entre el sector privado y la llamada economía social (Amin, 2009; Levi \& Davis, 2008; Tortia, 2009). Dichas organizaciones, de naturaleza híbrida (empresas con ánimo de lucro pero orientadas socialmente; Birchall, 2003; Hoffmann, 2006; Levi \& Davis, 2008), pueden tomar diferentes formas en cuanto a su estructura de propiedad, gerencia y razón social (Ridley-Duff \& Bull, 2011). Lo anterior en función de las diferentes legislaciones nacionales. A pesar de que en Colombia no existe una normativa que regule las empresas de naturaleza híbrida, algunas empresas con estructura de cooperativa llevan décadas actuando como empresas con ánimo de lucro e inspiradas por una misión social fundamental.

A nivel internacional, las empresas cooperativas de naturaleza híbrida han tomado diferentes nombres. Su actividad en países como Italia, Bélgica, Inglaterra, Francia, España y Portugal está bien documentada. De la misma manera, algunos estudios han explorado ciertos componentes psicológicos que se desprenden de su actividad cooperativa híbrida (Mosca, Musella, \& Pastore, 2008, Ohana \& Meyer, 2010; Tortia, 2009, 2008). Algunos académicos indican que uno de los principales retos de estas empresas es el de gestionar de manera adecuada sus recursos humanos (Ohana \& Meyer, 2010; Royce, 2007). Dado que estas organizaciones compiten en el mercado laboral, y que en muchas ocasiones se ven obligadas a ofrecer sueldos por debajo de aquellos que ofrecen las empresas con ánimo de lucro (Amin, 2009; Mosca et al., 2008), uno de sus principales retos que deben enfrentar consiste en retener sus recursos humanos calificados (Tortia, 2009). A esto se suma el hecho de que, durante las últimas décadas, el lazo que establecen los trabajadores con las empresas de tipo cooperativo se está debilitando (Fulton, 1999). En este sentido, la respuesta de la investigación sobre los recursos humanos ha sido igualmente insuficiente. El lazo de los trabajadores con las empresas cooperativas permanece relativamente inexplorado (Tuomine \& Jussila, 2010).

Ciertos estudios han abarcado la problemática de la retención de los recursos humanos en empresas cooperativas de naturaleza mixta. Por ejemplo, Ohana y Meyer (2010) investigaron la influencia que algunas variables relacionadas con la justicia organizacional tienen sobre la satisfacción en el trabajo y las intenciones de dejar la organización. Por su parte, Tortia $(2009,2008)$ encontró evidencia empírica del efecto que ejerce las percepciones de justicia organizacional sobre las intenciones de permanecer en la organización. Ahora bien, las investigaciones mencionadas hasta este punto han sido realizadas en el contexto europeo, mientras que sus resultados dejan en suspenso los mecanismos psicológicos que llevan a los trabajadores a permanecer o a dejar las empresas cooperativas de naturaleza híbrida. Hasta el momento, solo pocas investigaciones empíricas sobre la relación que establecen los trabajadores con las cooperativas latinoamericanas de naturaleza híbrida han sido publicadas (Román-Calderón, Battistelli, \& Férrieux, 2013). Hasta donde sabemos, ningún estudio empírico generalizable sobre las intenciones de dejar la organización en este contexto organizacional y regional ha sido publicado.

Las intenciones de dejar la organización han sido uno de los principales focos de interés de los estudiosos de las organizaciones. El apoyo organizacional percibido ha sido una de las variables más estudiadas en tanto factor predictor de las intenciones de dejar la organización. Este concepto, que parte de la teoría del intercambio social y de la reciprocidad, y que hace referencia a la relación empleador-empleado, es definido por Eisenberger, Huntington, Hutchison, y Sowa (1986) como las creencias que tienen los trabajadores acerca de la medida en la cual la organización valora sus contribuciones y se preocupa por su bienestar. La investigación empírica ha demostrado que el apoyo organizacional percibido ejerce una influencia negativa significativa e indirecta sobre las intenciones de dejar la organización (Maertz, Griffeth, Campbell, \& Allen, 2007). Ahora bien, esta relación no ha sido estudiada en cooperativas latinoamericanas de naturaleza híbrida. Es de esperarse que esta relación se dé en dicho contexto dado el fuerte vínculo que une a los trabajadores con las cooperativas (Tuomine \& Jussila, 2010). Algunos investigadores han demostrado que la relación entre el apoyo organizacional percibido y las intenciones de dejar la organización están mediadas por el compromiso hacia la organización (Maertz, Griffeth, Campbell, \& Allen, 2007). Las tres variables así estudiadas se refieren en conjunto a la relación que el trabajador establece con la organización. De manera alternativa, en el presente estudio se pretende estudiar cómo la relación empleado-empleador influencia la relación de los trabajadores con su trabajo, y cómo esta a su vez afecta las intenciones de dejar la organización.

En el contexto de las cooperativas de trabajo asociado, tanto la relación del empleado con la organización, como la relación de este con su trabajo, merecen la mayor atención. La relación de los empleados con su trabajo ha sido estudiada desde distintas perspectivas. Uno de los conceptos utilizados para 
ello es la implicación en el trabajo. Esta es entendida como el grado en el cual una persona está ligada con la organización en la que trabaja (Crossley, Bennett, Jex, \& Burnfield, 2007). Según Crossley et al., (2007), la implicación en el trabajo se diferencia de constructos como el compromiso organizacional pues integra aspectos relacionados con el trabajo mismo. Mientras que el compromiso organizacional ha sido propuesto como mediador entre el apoyo organizacional y las intenciones de dejar la organización (Maertz et al., 2007), la implicación en el trabajo no ha sido estudiada en este rol; menos aun dentro de las empresas de naturaleza mixta.

Es de esperarse que una buena relación del empleado con la empresa desemboque en una relación positiva de este con su trabajo en ella. Si el trabajador siente que la empresa no valora sus contribuciones hacia ella, si él o ella no se sienten apoyados por la organización, él mismo o ella misma perderá interés en su trabajo en dicha organización. A su vez, puede esperarse que una relación positiva del empleado con su trabajo en la empresa lo lleve a pensar en seguir trabajando en la organización. En particular, la investigación empírica ha demostrado que la implicación en el trabajo predice las intenciones de dejar la organización (Mitchell, Holtom, Thomas, Sablynski, \& Erez (2001). A partir de estos antecedentes parten las primeras tres hipótesis del presente estudio.

$\mathrm{H}_{1}$ : El apoyo organizacional percibido tiene un efecto positivo sobre la implicación en el trabajo

H2: La implicación en el trabajo ejerce una influencia negativa sobre las intenciones de dejar la organización.

H3: Por medio de la implicación en el trabajo, el apoyo organizacional percibido tiene un efecto negativo indirecto sobre las intenciones de dejar la organización.

Además de las ideas que tienen los empleados acerca de la valoración que hacen las organizaciones acerca de su trabajo, ellos desarrollan puntos de vista acerca de su relación con sus supervisores o jefes inmediatos (Eisenberger, Stinglhamber, Vandenberghe, Sucharski, \& Rhoades, 2002). En otras palabras, por fuera de los lazos que los empleados establecen con las organizaciones, ellos generan vínculos con sus supervisores inmediatos. Aunque estos últimos pueden fungir como representantes de la organización, los empleados tienen la capacidad de establecer la diferencia entre los vínculos con cada uno de esos focos (Becker, 1992; Dirks \& Ferrin, 2002; Reichers, 1985). Existe evidencia que indica que la relación que se establece con el supervisor tiene una influencia sobre las intenciones de dejar la organización (Griffeth, Hom, \& Gaertner 2000; Maertz, Stevens, \& Campion, 2003; Payne \& Huffman, 2005).
Algunos estudios basados en los conceptos de intercambio líder-miembro soportan dicha evidencia (Wayne, Shore, \& Liden, 1997; Wayne, Shore, Bommer, \& Tetrick, 2002). En particular, Harris, Wheeler, y Kacmar (2011) demostraron que el intercambio líder-miembro influye sobre las intenciones de dejar la organización por medio de la implicación en el trabajo. Sin embargo, en dicho estudio no se cotejaron dichas relaciones con la influencia que, mediada por la implicación en el trabajo, ejerce el apoyo organizacional sobre las intenciones de dejar la organización. En el presente estudio, desarrollado en un contexto organizacional diferente, se realiza dicha comparación por medio de la validación de las siguientes hipótesis conjuntamente con la evaluación de las tres primeras hipótesis:

$\mathrm{H}_{4}$ : El intercambio líder miembro tiene un efecto positivo sobre la implicación en el trabajo.

$\mathrm{H}_{5}$ : El intercambio líder miembro tiene un efecto indirecto negativo sobre las intenciones de dejar la organización por medio de la implicación en el trabajo.

A excepción de las hipótesis que conciernen los efectos indirectos del apoyo organizacional percibido y del intercambio líder miembro sobre las intenciones de dejar la organización (h3 y h4), los supuestos del estudio están representados en el modelo de mediación total que se presenta en la figura 1. La validación del modelo de mediación total propuesto en este artículo responde a la indicación hecha por algunos académicos, según la cual, la modelación de los mecanismos causales que explican las relaciones entre diferentes predictores y las intenciones de dejar la organización, es una de las futuras direcciones de la investigación (Maertz \& Griffeth, 2004). La validación del modelo hipotético representado aquí abajo permite entender y sopesar los efectos que la relación con la organización empleadora y la relación con el líder ejercen sobre las intenciones de dejar la organización por parte de los trabajadores. Así, el presente artículo ofrece una aproximación explicativa acerca de los problemas de retención que se presentan en las empresas cooperativas colombianas de naturaleza mixta.

\section{Figura 1. Modelo hipotético de mediación total}

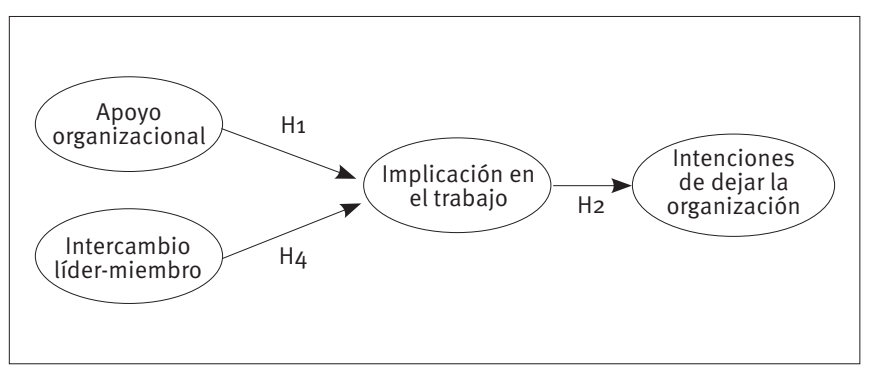




\section{MÉTODO}

A continuación se detallan los diferentes aspectos relacionados con el método utilizado para la realización del estudio descriptivo reportado en este artículo.

\section{Participantes y procedimiento}

La recolección de los datos se realizó por medio de un muestreo por conveniencia. En el estudio participaron voluntariamente 582 empleados de tres empresas cooperativas colombianas. De los 332 participantes efectivos un $93 \%$ fueron mujeres. La edad media de quienes respondieron el cuestionario fue de 28 años. En promedio, la permanencia de los empleados en las empresas era de 3 años. Estas empresas, que en promedio llevan 23 años de funcionamiento, comenzaron sus operaciones con el fin de integrar social y laboralmente mujeres afectadas por la violencia. De allí la distribución de datos dispar en términos de género. De los 582 cuestionarios suministrados en papel, 332 resultaron utilizables para los análisis efectuados en este estudio. Los cuestionarios que contenían datos faltantes no fueron tenidos en cuenta para los análisis. Por el contrario, los datos atípicos fueron mantenidos. Esta decisión se debió a que dichas observaciones no correspondían a errores en la transcripción de los datos sino a los puntajes asignados por los participantes. El cuestionario fue traducido al español y luego retraducido al inglés por traductores independientes. Lo anterior con el fin de asegurar una traducción fiel de las escalas de medición al castellano. Una prueba piloto del cuestionario fue realizada con la colaboración de 24 empleados de una de las cooperativas participantes. Como resultado de la prueba piloto, algunos ítems fueron descartados por dificultades para la comprensión. Este fue el caso de tres de los indicadores de la escala intercambio líder-miembro. Particularmente, aquellos ítems de la escala intercambio líder-miembro que preguntaban sobre el aprecio de los empleados por las competencias del líder.

\section{Instrumento de medición}

Los participantes respondieron a todos los enunciados del cuestionario utilizando una escala Likert de 5 puntos. La palabra organización fue reemplazada con el nombre cooperativa con el fin de adaptar el cuestionario al contexto organizacional bajo estudio.

El intercambio líder-miembro (LMX) fue medido utilizando una versión reducida de la escala desarrollada por Liden y Maslyn (1998). La sección del cuestionario que introdu- cía la escala LMX señalaba a los participantes: "Esta sección se refiere a su percepción sobre la relación con su jefe inmediato". Los siguientes son ejemplos de los ítems de la escala LMX utilizada: "Es muy satisfactorio trabajar con mi jefe inmediato"; "Estoy dispuesto a trabajar fuertemente por mi superior".

La versión reducida de 8 ítems del cuestionario de Eisenberger, Huntington, Hutchison y Sowa (1986) fue empleada para medir el apoyo organizacional percibido (APO). Los siguientes son ejemplos de los indicadores que conformaban la escala utilizada: "Mi cooperativa considera muy importantes mis metas y mis valores"; "En mi cooperativa las personas se preocupan por mi satisfacción en el trabajo"; "En mi cooperativa realmente se preocupan por mi bienestar".

Una versión de tres ítems de la escala desarrollada por Crossley, Bennett, Jex, y Burnfield, (2007) fue utilizada para evaluar la implicación en el trabajo (IT) de los participantes. La escala era presentada a los participantes con el siguiente encabezado: "Esta sección del cuestionario corresponde a su opinión respecto a su vida en general". Algunos ejemplos de los indicadores que componían la escala IT son: "Me siento cercano a esta cooperativa"; "Estoy demasiado bien integrado en esta cooperativa como para irme de ella".

Las intenciones de dejar la organización (IDO) fueron evaluadas por medio del instrumento desarrollado por Bentein, Vandenberg, Vandenberghe, y Stinglhamber, 2005. El encabezado que introducía los indicadores de esta escala rezaba: "En esta sección encontrará una serie de afirmaciones a propósito de su futuro laboral". Los ítems de la escala eran los siguientes: "Pienso a menudo en dejar la cooperativa para la cual trabajo" y "Tengo intenciones de buscar trabajo en otra organización durante el año que viene".

\section{Análisis}

El paquete estadístico M-plus versión 6.11 (Muthén \& Muthén, 2010) fue utilizado para el análisis de datos. Un análisis factorial confirmatorio para verificar las propiedades psicométricas del instrumento se hizo siguiendo la técnica de modelización de ecuaciones estructurales. En este caso, se utilizó el método de estimación de máxima verosimilitud. Fueron calculados los coeficientes de asimetría y curtosis para evaluar en qué medida las variables observadas se ajustaban al supuesto de normalidad que presupone el método de estimación. Aunque las variables observadas no cumplen cabalmente con el supuesto de normalidad, solo en el caso de una de las variables observadas del factor implicación en el trabajo el valor límite de la curtosis fue levemente superado (> 3; Kline, 2011). 
Para evaluar la adecuación del análisis factorial confirmatorio a los datos se utilizaron los siguientes índices: $x^{2} / d f$, RMSEA, TLI y SRMR. Los valores límites utilizados como referentes para verificar el ajuste general de los modelos estructurales son aquellos sugeridos por Schreiber, Nora, Stage, Barlow, y King (2006). Dado que el alfa de Cronbach no es normalmente utilizado en análisis confirmatorios, otras estadísticas fueron utilizadas para valorar la confiabilidad del instrumento (Hair, Anderson, Tatham, \& Black, 2008). El índice de confiabilidad compuesta (CR) mide la consistencia interna de los constructos presentes en el análisis factorial confirmatorio. El umbral para aceptar la hipótesis de confiabilidad de cada uno de los constructos es $\geq 0,7$ (Hair et al., 2008). Ahora bien, el CR no es suficiente para concluir sobre la precisión con la cual los indicadores miden el constructo latente. Por lo tanto se calculó la varianza extraída (AVE) para cada uno de ellos. Esta mide la cantidad de varianza explicada por el constructo, en contraste con la varianza generada por los errores de medición. El AVE provee evidencia sobre la validez convergente de los constructos utilizados. Si AVE <0,5, tanto la validez de cada uno de los indicadores como del constructo es cuestionable. Adicionalmente si el coeficiente AVE de dos constructos es mayor al cuadrado de la correlación entre ellos, la validez discriminatoria de estos está garantizada (Fornell \& Larcker, 1981).

Posteriormente fue puesto a prueba el modelo de mediación total constituido por las hipótesis de estudio. Dicho modelo fue probado por medio de la modelación de ecuaciones estructurales. Este tipo de modelación permite examinar relaciones complejas entre variables latentes. Los índices de adecuación a la muestra y los valores límites utilizados fueron los mismos usados para validar el análisis factorial confirmatorio. Los efectos indirectos del apoyo organizacional percibido y del intercambio líder miembro fueron evaluados por medio del test Sobel (Sobel, 1982) y de intervalos de confianza generados por re-muestreo Bootstrap.

\section{RESULTADOS}

El análisis factorial confirmatorio corrobora la estructura factorial prevista. La solución con los cuatro factores teóricos obtuvo unos índices de adecuación general aceptables $\left(x^{2}=300,58_{\text {df113 }}\right.$, $p<0,05 ;$ RMSEA $=0,07 ; \mathrm{TLI}=0,93 ;$ SRMR $=0,04)$. Como puede observarse en la tabla 1, todos los constructos poseen índices de confiabilidad satisfactorios. Adicionalmente, se obtuvo evidencia acerca de la validez convergente de los factores encontrados por medio del análisis confirmatorio. Los porcentajes de varianza extraída superaron el valor límite $(0,5)$.

\section{TABLA 1. Análisis factorial confirmatorio - Medidas de validez convergente (AVE) ${ }^{\text {a }}$ y confiabilidad de los factores (CR)}

\begin{tabular}{c|c|c}
\hline Variable Latente & $\begin{array}{c}\text { Varianza extraída } \\
\text { (AVE) }\end{array}$ & $\begin{array}{c}\text { Confiabilidad } \\
\text { compuesta (CR) }\end{array}$ \\
\hline AOP & $66 \%$ & 0,92 \\
\hline LMX & 60 & 0,90 \\
\hline IT & 56 & 0,79 \\
\hline IDO & 60 & 0,75 \\
\hline
\end{tabular}

Nota: $\mathrm{n}=332{ }^{\text {a }}$ siglas en inglés

Igualmente, el coeficiente de varianza extraída AVE de todos los constructos superó el cuadrado de las correlaciones (ver tabla 2) de cada uno de estos con respecto a los demás factores. De allí se pudo concluir validez discriminante para todas las variables latentes.

\section{TABLA 2. Correlaciones de Pearson entre las variables latentes.}

\begin{tabular}{c|c|c|c}
\hline Variable latente & $\mathbf{1}$ & $\mathbf{2}$ & $\mathbf{3}$ \\
\hline AOP & & & \\
\hline LMX & 0,63 & & \\
\hline IT & 0,64 & 0,40 & \\
\hline IDO & $-0,33$ & $-0,25$ & $-0,48$ \\
\hline
\end{tabular}

Nota: $n=332$

Tres ítems de la escala de intercambio líder-miembro debieron ser retirados. Las saturaciones de estos ítems presentaron valores atípicos. Una explicación para ello es que dos de estos indicadores eran los únicos que se referían a la posibilidad de establecer relaciones amistosas con el superior. El ítem restante se refería más al esfuerzo que harían los trabajadores a favor del grupo, lo cual desplaza el foco de atención del líder y lo ubica en el grupo.

El modelo de mediación hipotético (modelo 1) obtuvo unos índices satisfactorios de adecuación a la muestra (ver tabla 3). Estos resultados permiten validar las hipótesis h1 y h2. Adicionalmente, el efecto indirecto del apoyo organizacional percibido sobre las intenciones de dejar la organización resultó negativo ( $\beta=-0,31 p<.001)$ y significativo al $99 \%$ dada la ausencia de cero en el intervalo de confianza bootstrap al 99\% (-0,44 $-0,18)$. Este último hallazgo permite validar la hipótesis h3, según la cual, el apoyo organizacional, por medio de la implicación en el trabajo, tiene un efecto indirecto negativo sobre las intenciones de dejar la organización. Contrariamente, el efecto indirecto del intercambio líder-miembro no resultó significativo $(\beta=-0,004 p=0,91)$. El resultado del test Sobel fue confirmado por la presencia de cero en el intervalo bootstrap al 99\% (-0,09 $0,08)$. De allí que no haya sido posible, siguiendo el modelo hipotético inicial, validar las hipótesis $\mathrm{H}_{4}$ y $\mathrm{H}_{5}$. 
TABLA 3. Coeficientes de ajuste de los modelos de regresión y prueba Satorra-Bentler

\begin{tabular}{|c|c|c|c|c|c|}
\hline Modelo de regresión & $x^{2}$ & $\mathrm{df}$ & RMSEA & TLI & SRMR \\
\hline 2. Mediación Parcial LMX->|DO & 300,58 & 114 & 0,07 & 0,93 & 0,04 \\
\hline 4. Mediación LMX->AOP->AOP->|DO & 301,43 & 116 & 0,07 & 0,94 & 0,04 \\
\hline Comparación & & $x^{2 \text { diff }}$ & df & $\mathrm{p}$ valor & \\
\hline 1 versus 3 & & 0,30 & 1 & $>0,01$ & Modelo 1 \\
\hline 1 versus 4 & & 0,01 & 1 & $>0,01$ & Modelo 4 \\
\hline
\end{tabular}

Nota: $n=332$

Dada la posibilidad de que el intercambio líder-miembro tuviese un efecto directo sobre las intenciones de dejar la organización, un segundo modelo de regresión (modelo 2) fue puesto a prueba. En el modelo 2, se agregó un parámetro que unía el intercambio líder-miembro en una relación causal. La adición de este nuevo parámetro estuvo sustentada en los hallazgos de Maertz et al., (2007). Según estos autores, la relación con el supervisor o jefe inmediato tendría un efecto directo sobre las intenciones de dejar la organización. La adición de este nuevo parámetro no arrojó resultados satisfactorios. De un lado, por medio del test Satorra-Bentler ( $x^{\text {2diff }}$, que permite realizar una comparación entre modelos estructurales anidados (Kline, 2011), el modelo 1 fue preferido por encima del modelo 2 (ver tabla 3). Resultando el test Satorra-Bentler ( $\left.x^{\text {2diff }}\right)$ no significativo, se abrió la posibilidad de elegir el modelo menos complejo (mayor número de grados de libertad), en este caso, el modelo 1 . El rechazo del modelo 2 fue soportado por el hecho de que el parámetro agregado, aquel que sugería que el intercambio líder-miembro ejercía un efecto directo sobre las intenciones de dejar la organización, no resultó significativo $(\beta=-0,07 p=0,36)$. Con el fin de verificar la exis- tencia de una mediación total entre el apoyo organizacional percibido y las intenciones de dejar la organización, se agregó al modelo 1 un parámetro directo desde la primera de estas variables hasta la variable criterio (modelo 3; tabla 3). Dicho parámetro no resultó significativo $(\beta=-0,07 p=0,59)$. Adicionalmente, dada la no significancia del test Satorra-Bentler ( $\left.x^{2 \text { diff }}\right)$, el modelo 1 fue preferido sobre el modelo 3.

Finalmente, siguiendo la literatura sobre el apoyo organizacional percibido y el apoyo del superior inmediato, fue probado un cuarto modelo (modelo 4; ver figura 2). Algunas investigaciones han mostrado que el efecto de la relación con el supervisor inmediato sobre el abandono de la organización está mediado por el apoyo organizacional percibido (Eisenberger et al., 2002; Rhoades, Eisenberger, \& Armeli, 2001). Teniendo en cuenta estos antecedentes teóricos, el hecho que el intercambio líder miembro no mostró un efecto ni directo ni indirecto sobre las intenciones de dejar la organización (ver modelo hipotético), y que además el intercambio con el supervisor mostraba una correlación alta con el apoyo organizacional percibido (ver tabla 2), el intercambio líder-miembro se propuso como variable independiente en el modelo 4.

\section{Figura 2. Modelo de regresión retenido (modelo 4)}

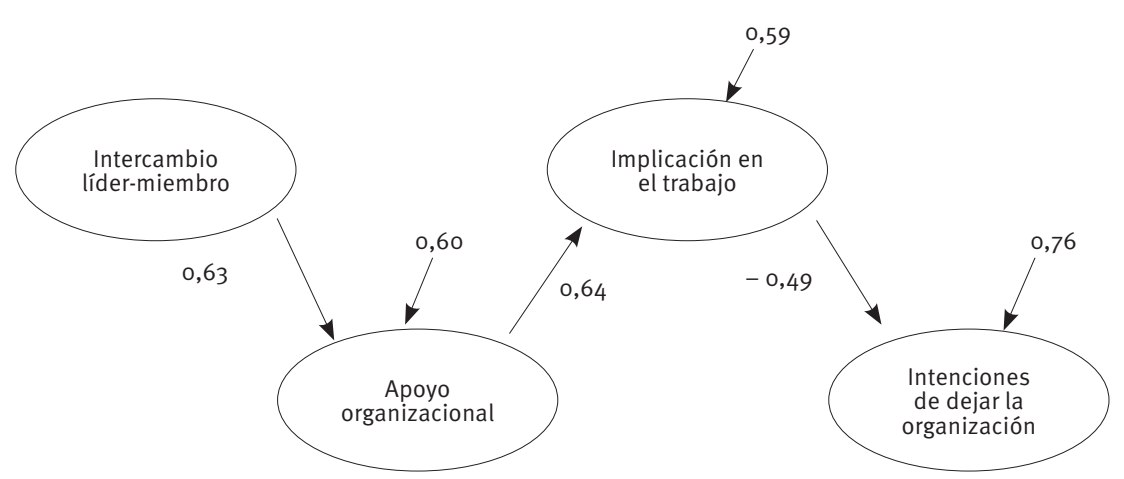

Nota: Todos los coeficientes estandarizados significativos al p<0,01. El modelo explica una proporción significativa de la varianza del apoyo organizacional percebido $\left(R^{2}=0,40\right.$, p<o,01), de la varianza de la implicación en el trabajo $\left(R^{2}=0,41, p<0,01\right)$ y de la varianza de las intenciones de dejar la organización $\left(R^{2}=0,24\right.$, p<.01). 
El modelo 4 obtuvo unos índices satisfactorios de adecuación a la muestra. Como puede observarse en la tabla 3, el test Satorra-Bentler ( $\mathrm{x}^{\text {2diff }}$ ) por medio del cual se compararon los modelos 1 y 4, resultó no significativo. Por lo tanto, el modelo más simple (con mayores grados de libertad) puedo ser seleccionado por encima del modelo más complejo. Igualmente, el coeficiente TLI jugó a favor de la elección del modelo 4, dado que su valor se acercaba más a la unidad (ver tabla 3). Para sustentar la elección del modelo 4, el efecto indirecto del intercambio líder-miembro sobre las intenciones de dejar la organización fue calculado. El efecto indirecto resultó ser moderado y significativo $(\beta=-0,20 p<0,001)$. La significancia de este efecto indirecto se vio confirmada por la ausencia de cero en el intervalo de confianza bootstrap al 99\% $(-0,34-0,12)$. A partir de este hallazgo es posible validar parcialmente la h $_{5}$, según la cual, el intercambio líder-miembro tiene un efecto indirecto negativo significativo sobre las intenciones de dejar la organización de los trabajadores de las cooperativas mixtas. La validación de esta hipótesis es parcial pues, contrariamente el modelo hipotético propuesto inicialmente, en el modelo 4 a la implicación en el trabajo se le suma el apoyo organizacional percibido como mediador. Adicionalmente, los resultados positivos del modelo 4 permiten validar parcialmente la h4. Se trata igualmente de una validación parcial pues los resultados definitivos indican que el efecto positivo del intercambio líder-miembro sobre la implicación en el trabajo no es directo, como se había supuesto inicialmente, sino indirecto $(\beta=0,40 p<0,001)$. La significancia de este efecto positivo indirecto se ve confirmada por la ausencia de cero en el intervalo de confianza bootstrap al 99\% (0,22 0,44).

\section{DISCUSIÓN}

La investigación presentada en el presente artículo tenía como objetivo analizar si el apoyo organizacional percibido y el intercambio líder-miembro de los trabajadores de empresas cooperativas de naturaleza mixta, ejercen una influencia sobre las intenciones de dejar la organización por parte de ellos. El estudio de los antecedentes de esta última variable es de especial importancia en organizaciones de naturaleza híbrida, pues ellas enfrentan problemas de reclutamiento y retención de personal, y en muchas ocasiones encuentran dificultades para ofrecer los mismos salarios que sus competidoras con ánimo de lucro (Amin, 2009; Mosca et al., 2008). Es por ello que para las organizaciones de naturaleza híbrida, el conocimiento de los mecanismos por medio de los cuales variables no monetarias influencian las intenciones de dejar la organización, es de gran importancia. El análisis de los datos obtenidos en 3 cooperativas colombianas de naturaleza híbrida ofreció resultados in- éditos con respecto a algunos antecedentes no monetarios de las intenciones de dejar la empresa en el contexto organizacional en mención.

El estudio partió del supuesto de que la relación líder-miembro tiene efectos independientes sobre las intenciones de dejar la organización (Wayne et al., 1997; Wayne et al., 2002). Así, se propuso un modelo en el cual se proponía un modelo en el cual la relación líder-miembro y el apoyo organizacional percibido actuaban ambas como variables independientes. Contrariamente a las hipótesis, la modelación por ecuaciones estructurales indicó que, al igual como lo sugieren ciertos estudios precedentes (Eisenberger et al., 2002; Rhoades et al., 2001), el efecto que tiene la relación con el superior inmediato sobre las intenciones de dejar la organización está mediado por el apoyo organizacional. Adicionalmente, los resultados presentados en este artículo sugieren un segundo mediador entre el intercambio líder-miembro y las intenciones de dejar la organización: la implicación en el trabajo. Este resultado tiene sentido si se considera que, para los trabajadores de la empresas cooperativas de naturaleza mixta, el trabajo tiene un significado especial que sobrepasa la retribución monetaria (Mosca, MuseIla \& Pastore, 2007; Tortia, 2009, 2008). En palabras de Garcia (1981), en las asociaciones cooperativas la noción de producción y de trabajo presupone características meta-económicas. Por lo tanto, es plausible suponer que cuando estos trabajadores están implicados en su trabajo, sus intenciones de abandonar la empresa decrecen. De hecho, esta investigación provee evidencia empírica que soporta dicha suposición.

\section{Implicaciones teóricas y prácticas}

Desde el punto de vista teórico, esta investigación es novedosa dada la inclusión de la implicación en el trabajo como variable mediadora de los efectos de la relación líder-miembro sobre las intenciones de dejar la organización. De manera diferente a otras investigaciones, donde los efectos del apoyo organizacional y de la relación con el supervisor sobre las intenciones de dejar la organización resultó mediada por actitudes de los trabajadores hacia la empresa (Maertz et al., 2007), este estudio incluye como mediadora una variable cuyo foco es el trabajo y no exclusivamente la organización. En resumen, esta investigación sugiere que dentro de las organizaciones cooperativas de naturaleza mixta una buena relación con el supervisor tiene un impacto indirecto negativo sobre las intenciones de dejar la organización. Esta influencia se da por la idea de sentirse apoyado por la organización y de la implicación de los trabajadores en el trabajo. Esto es, ante una mejor relación con el jefe inmediato, mayor percepción de apoyo organizacional, mayor impli- 
cación en el trabajo y, en consecuencia, menores intenciones de dejar la cooperativa por parte de los trabajadores de cooperativas híbridas. Así, el presente artículo contribuye a la teoría del intercambio líder-miembro y a la literatura sobre las organizaciones de naturaleza híbrida mostrando uno de los mecanismos psicológicos por medio de los cuales la relación con el supervisor y/o jefe inmediato influye sobre las intenciones de dejar la organización.

Los hallazgos presentados aquí tienen las siguientes implicaciones prácticas. De un lado, se suma a las evidencias empíricas que sugieren a los administradores de empresas cooperativas de naturaleza híbrida, la necesidad de prestar mayor atención a los componentes no-monetarios como antecedentes del bienestar en el trabajo y de las intenciones de permanecer en la organización (Tortia, 2009, 2008). Los aspectos no-monetarios, o meta económicos, son fundamentales para la gestión y desarrollo de los recursos humanos de empresas con estructuras cooperativas (Garcia, 1981; Ohana \& Meyer, 2010). La consideración de las relaciones aquí expuestas entre el intercambio líder-miembro, el apoyo organizacional y la implicación en el trabajo, puede ayudar a los administradores de las empresas cooperativas mixtas a combatir los problemas de reclutamiento y retención de personal a los que se ven enfrentadas dichas organizaciones. Finalmente, el presente artículo se ofrece como referencia a profesionales que dirigen empresas cooperativas latinoamericanas y colombianas de naturaleza mixta. La investigación acerca de los recursos humanos de este tipo de organizaciones en Latinoamérica es escasa. Si bien la literatura internacional relaciona prácticas organizacionales cooperativas de naturaleza mixta en países como Brasil y Argentina (Amin, 2009; Kerlin, 2010; Seelos \& Mair, 2005), poca evidencia empírica generalizable se ha publicado sobre elementos que permitan un mejor manejo y desarrollo de los recursos humanos de empresas latinoamericanas y colombianas de este tipo.

\section{Limitaciones y direcciones para la investigación}

El estudio presentado en este artículo tuvo un diseño transversal. Dicho diseño impide extraer resultados concluyentes acerca de las relaciones causales entre las variables estudiadas. Para verificar dichas relaciones explicativas, futuras investigaciones deberán considerar la posibilidad de implementar diseños longitudinales. Otra de las limitaciones del estudio consiste en que no se tuvo la ocasión de agrupar los datos de acuerdo a los grupos de trabajo y de los supervisores correspondientes. Esto impidió la ejecución de análisis multinivel. Por lo tanto, futuras investigaciones deberán concentrarse en la recolección de futuros datos agregados y los análisis multinivel correspondientes; más aun cuando se estudian variables que como el intercambio líder-miembro tienen una naturaleza grupal.

Una de las pretensiones del presente artículo es la de incentivar los estudiosos de las organizaciones a realizar estudios sobre problemáticas que enfrentan las cooperativas latinoamericanas de naturaleza mixta. Estas organizaciones son una de las claves para la solución de los problemas socio-económicos que enfrentan las comunidades (Birchall, 2003; Seelos \& Mair, 2005). Dados los numerosos problemas que a este nivel enfrentan los países latinoamericanos, los estudios que permitan la reflexión en torno a la administración de dichas empresas merecen la mayor atención por parte de los académicos de la región.

\section{REFERENCIAS}

Amin, A. (2009). Extraordinarily ordinary: working in the social economy. Social Enterprise Journal, 5(1), 30-49.

Becker, T. E. (1992). Foci and bases of commitment: are they distinctions worth making? Academy of Management Journal, 35(1), 232-244.

Bentein, K., Vandenberg, R., Vandenberghe, C., \& Stinglhamber, F. (2005). The role of change in the relationship between commitment and turnover: a latent growth modeling approach. Journal of Applied Psychology, 90(3), 468-482.

Birchall, J. (2003). Rediscovering the Cooperative Advantage: poverty reduction through self-help. Geneva: International Labour Office.

Crossley, C. D., Bennett, R. J., Jex, S. M., \& Burnfield, J. L. (2007). Development of a global measure of job embeddedness and integration into a traditional model of voluntary turnover. Journal of Applied Psychology, 92(4), 1031-1042.

Dirks, K. T. \& Ferrin, D. L. (2002). Trust in leadership: meta-analytic findings and implications for research and practice. Journal of Applied Psychology, 87(4), 611-628.

Eisenberger, R., Huntington, R., Hutchison, S., \& Sowa, D. (1986). Perceived organizational support. Journal of Applied Psychology, 71(3), 500-507.

Eisenberger, R., Stinglhamber, F., Vandenberghe, C., Sucharski I. L, \& Rhoades, L. (2002). Perceived supervisor support: contributions to perceived organizational support and employee retention. Journal of Applied Psychology, 87(3), 565-573.

Fornell, C. \& Larcker, D. F. (1981). Evaluating structural equation models with unobservable variables and measurent error. Journal of Marketing Research, 18(3), 39-50.

Fulton, M. (1999). Cooperatives and Member Commitment. The Finnish Journal of Business Economics, 48(4), 418-437.

Garcia, R. M. (1981). Os requisitos de um programa de treinamento de cooperativas. RAE-Revista de Administração de Empresas, 21(1), 39-45. 
Griffeth, R. W, Hom, P. W, \& Gaertner, S. (2000). A meta-analysis of antecedents and correlates of employee turnover: updated moderator tests, and research implications for the next millennium. Journal of Management, 26(3), 463-488.

Hair, J., Anderson, R. E., Tatham, R., \& Black, W. (2008). Multivariate data analysis. New Jersey: Prentice Hall.

Hoffmann, E. A. (2006). The ironic value of loyalty: dispute resolution strategies in worker cooperatives and conventional organizations. Nonprofit Management and Leadership, 17(2), 163-177.

Harris, K. J., Wheeler, A. R., \& Kacmar, K. M. (2011). The mediating role of organizational job embeddedness in the LMX-outcomes relationships. The Leadership Quarterly, 22(2), 271-281.

Kerlin, J. A. (2010). A comparative analysis of the global emergence of social enterprise. Voluntas: International Journal of Voluntary and Nonprofit Organizations, 21(2), 162-179.

Kline, R. B. (2011). Principles and practices of structural equation modeling. New York: The Guilford Press.

Levi, Y. \& Davis, P. (2008). Cooperatives as the "enfants terribles" of economics: some implications for the social economy. The Journal of Socio-Economics, 37(6), 2178-2188.

Liden, R. C. \& Maslyn, J. M. (1998). Multidimensionality of leader-member exchange: an empirical assessment through scale development. Journal of Management, 24(1), 43-72.

Maertz, Carl P., Griffeth, R. W., Campbell, N. S., \& Allen, D. G. (2007). The effects of perceived organizational support and perceived supervisor support on employee turnover. Journal of Organizational Behavior, 28(8), 1059-1075.

Maertz C. P., \& Griffeth, R. W. (2004). Eight motivational forces and voluntary turnover: a theoretical synthesis with implications for research. Journal of Management, 30(5) 667-683.

Maertz, C. P., Stevens, M. J., \& Campion, M. A. (2003). A turnover mod el for the Mexican maquiladoras. Journal of Vocational Behavior, 63(1), 111-135.

Mosca, M., Musella, M., \& Pastore, F. (2007). Relational goods, monitoring and non-pecuniary compensations in the nonprofit sector: the case of the Italian social services. Annals of Public and Cooperative Economics, $78(1)$, 57-86

Mosca, M., Musella, M., \& Pastore, F. (2008). Relational goods, monitoring and non-pecuniary compensations in the nonprofit sector: the case of the Italian social services. Annals of Public and Cooperative Econom ics, $78(1), 57-86$

Ohana, M., \& Meyer, M. (2010). Should I stay or should I go now? Investigating the intention to quit of the permanent staff in social enterprises. European Management Journal, 28(6), 441- 454.

Payne, S. C. \& Huffman, A. H. (2005). A longitudinal examination of the influence of mentoring on organizational commitment and turnover. Academy of Management Journal, 48(1), 158-168.
Mitchell, T. R., Holtom, B. C., Lee, T. W., Sablynski, C. J., \& Erez, M. (2001). Why people stay: using job embeddedness to predict voluntary turnover. Academy of Management Journal, 44(6), 1102-1121.

Muthén, L. K. \& Muthén, B. O. (2010). Mplus user's guide. Los Angeles, CA: Muthén \& Muthén.

Reichers, A. (1985). A review and reconceptualization of organizational commitment. Academy of Management Review, 10(3), 465-476.

Rhoades, L., Eisenberger, R., \& Armeli, S. (2001). Affective commitment to the organization: the contribution of perceived organizational support. Journal of Applied Psychology, 86(5), 825-836.

Ridley-Duff, R. \& Bull, M. (2011). Understanding social enterprise: theory and practice. London: Sage.

Román-Calderón, J. P., Battistelli, A., \& Ferrieux, D. (2013). Mesure de l'invariance d'une echelle de contrat psychologique ideologique. Collection Psychologie du Travail. Paris: l'Harmattan.

Royce, M. (2007). Using human resource management tools to support social enterprise: emerging themes from the sector. Social Enterprise Journal, 3(1), 10-19.

Schreiber, J. B., Nora, A., Stage, F. K., Barlow, E. A., \& King, J. (2006). Reporting structural equation modeling and confirmatory factor analysis results: a review. The Journal of Educational Research, 99(6), 323-338.

Seelos, C. \& Mair, J. (2005). Social entrepreneurship: creating new business models to serve the poor. Business Horizons, 48(3), 241-246.

Sobel, M. E. (1982). Asymptotic confidence intervals for indirect effects in structural equation models. In Leinhardt, S. (ed). Sociological methodology 1982. Washington, DC: American Sociological Association, 1982, pp. 290-312.

Tortia, E. (2009). Perceived fairness and worker well-being in public, for-profit and nonprofit firms: evidence from the Italian social service sector. In Destefanis, S. \& Mussella, M. (eds.). Paid and Unpaid Labour in the Social Economy. Heidelberg: Physica-Verlag HD, pp. 97-114.

Tortia, E. (2008). Worker well-being and perceived fairness: survey-based findings from Italy. The Journal of Socio-Economics, 37(5), 2080-2094.

Tuomine, P., \& Jussila, I. (2010). The individual psychological impact of ownership in co-operatives. The International Journal of Co-operative Management, 5(1), 23-33.

Wayne, S. J., Shore, L. M., Bommer, W. H., \& Tetrick, L. E. (2002). The role of fair treatment and rewards in perceptions of organizational support and leader-member exchange. Journal of Applied Psychology, 87(3), 590-598.

Wayne, S. J., Shore, L. M., \& Liden, R. C. (1997). Perceived organizational support and leader-member exchange: a social exchange perspective. Academy of Management Journal, 40(1), 82-111. 